12.1

\title{
Определение энергии и флюенсов протонов, коллективно ускоренных в ускорителе с диодом Люса
}

\author{
(С) В.А. Рыжков, Г.Е. Ремнев, М.В. Журавлев, И.Н. Пятков, В.С. Лопатин
}

Исследовательская школа фризики высокоэнергетических процессов, Томский политехнический университет, Томск, Россия

E-mail: slavar@fibrtec.com

Поступило в Редакцию 25 февраля 2019г.

В окончательной редакции 5 апреля 2019г.

Принято к публикации 19 апреля 2019 г.

Пластины карбида бора $\mathrm{B}_{4} \mathrm{C}$ использованы в качестве термостойких мишеней для определения энергии и флюенсов протонов, коллективно ускоренных в ускорителе с диодом Люса, посредством контроля гаммарадиоактивности радионуклидов ${ }^{7} \mathrm{Be} и{ }^{13} \mathrm{~N}$, наведенных в ядерных реакциях ${ }^{10} \mathrm{~B}(p, \alpha){ }^{7} \mathrm{Be}$ и ${ }^{12} \mathrm{C}(p, \gamma){ }^{13} \mathrm{~N}$. Диод Люса с цилиндрическим вольфрамовым катодом и анодом в виде полиэтиленовой шайбы генерировал электронный пучок с длительностью до $100 \mathrm{~ns}$ и током до $30 \mathrm{kA}$ при напряжении около $250 \mathrm{kV}$. Предложенная методика позволяет контролировать энергию протонов в пределах $500-1500 \mathrm{keV}$ и флюенсы до $5 \cdot 10^{13}$ protons $/\left(\mathrm{cm}^{2} \cdot\right.$ pulse $)$.

Ключевые слова: радиоактивность, гамма-спектрометрия, импульсный пучок частиц, карбид бора.

DOI: 10.21883/PJTF.2019.14.48020.17752

Разработка ускорителей с диодом Люса требует простой и надежной методики контроля энергии и флюенсов коллективно ускоренных ионов с энергией от 500 до $1500 \mathrm{keV}$ [1]. При условии, что облученные мишени не эродируют под действием мощных импульсных ионных пучков (МИИП), протонно-индуцированная активация выглядит простой и надежной методикой для оценки флюенсов и энергии таких протонов. В этом плане пластины карбида бора $\mathrm{B}_{4} \mathrm{C}$ с температурой плавления около $3250^{\circ} \mathrm{C}$ представляют несомненный интерес для осуществления радиоактивационного контроля энергии и флюенсов протонов за счет одновременной регистрации гамма-активности радионуклидов ${ }^{7} \mathrm{Be}$ и ${ }^{13} \mathrm{~N}$, наведенных в мишенях по ядерным реакциям ${ }^{10} \mathrm{~B}(p, \alpha)^{7} \mathrm{Be}$ и ${ }^{12} \mathrm{C}(p, \gamma){ }^{13} \mathrm{~N}$ соответственно. Выход первой реакции быстро растет в интервале энергий протонов от 500 до $1500 \mathrm{keV}$ [2], тогда как выход второй реакции в этом интервале едва увеличивается [3]. В результате отношение этих выходов быстро нарастает с увеличением энергии протонов, что позволяет определять не только их энергию, но и флюенсы. Возможная эрозия поверхности мишеней может приводить к занижению оценки энергии протонов при их энергии существенно больше энергии первого резонанса реакции ${ }^{12} \mathrm{C}(p, \gamma)^{13} \mathrm{~N}$, т.е. $459 \mathrm{keV}$. Это происходит из-за того, что ${ }^{7} \mathrm{Be}$, наведенный в мишени с большей концентрацией у самой поверхности, испаряется в намного большей степени, чем ${ }^{13} \mathrm{~N}$, локализованный в данном случае преимущественно в узком слое на глубине мишени. Поэтому основным ограничением указанной методики в интервале энергий коллективно ускоренных протонов 500-1500 keV является максимальный флюенс ускоренных ионов.

В настоящей работе приведены результаты тестирования специальной геометрии диода Люса в импульсном электронном ускорителе ТЕМП, который в согласованном режиме характеризуется ускоряющим напряжением $200 \mathrm{kV}$, электронным током до $40 \mathrm{kA}$ и длительностью импульса напряжения на полувысоте $90 \mathrm{~ns}$ [4]. В тестируемой геометрии диода Люса импеданс ускоряющей системы был выше, чем в согласованном режиме, и в результате напряжение достигало $250 \mathrm{kV}$, а электронный ток был ниже (около $30 \mathrm{kA}$ ). В этой геометрии диода в качестве катода использован цилиндрический вольфрамовый стержень диаметром $4 \mathrm{~mm}$ и длиной $15 \mathrm{~mm}$, а в качестве анода - шайбы из полиэтилена высокого давления с толщиной $8 \mathrm{~mm}$ и диаметром отверстия $10 \mathrm{~mm}$. Катод соосно углублен в отверстие анода на $3 \mathrm{~mm}$. Полиэтиленовые (ПЭ) и медные заземленные полые усеченные конусы длиной $80 \mathrm{~mm}$ были установлены на поверхности анода со стороны заанодного пространства в соответствии с рекомендациями [4]. Согласно [5], размещение подобных элементов приводило к увеличению энергии коллективно ускоренных ионов и стабилизации энергии и флюенсов ионов от импульса к импульсу. Расстояние между анодом и дисками $\mathrm{B}_{4} \mathrm{C}$ мишени с диаметром $15 \mathrm{~mm}$ и толщиной 1-2 $\mathrm{mm}$ было $90 \mathrm{~mm}$ во всех опытах, кроме эксперимента № 3, где итоговое расстояние от анода до мишени составляло $130 \mathrm{~mm}$ (см. таблицу). Диски $\mathrm{B}_{4} \mathrm{C}$ были размещены соосно с катодом и анодом и приклеены двусторонним коммерческим полипропиленовым скотчем Profitto $(80 \mu \mathrm{m})$ к медному держателю. Передняя поверхность дисков $\mathrm{B}_{4} \mathrm{C}$ была зеркально отполирована для осуществления визуального контроля за возможной эрозией поверхности при воздействии МИИП. Видимой эрозии не было обнаружено во всех опытах, но возможность уноса тонкого приповерхностного слоя $\left(\sim 0.3 \mathrm{mg} / \mathrm{cm}^{2}\right)$ за импульс не была исключена для опыта № 2, где наблюдалась небольшая убыль веса после облучения. Использование 
Флюенсы и энергии коллективно ускоренных протонов

\begin{tabular}{c|l|c|c|c|c|c|c|c|c}
\hline $\begin{array}{c}\text { Номер } \\
\text { опыта }\end{array}$ & $\begin{array}{c}Z \text { (анод-мишень }), \\
\mathrm{mm}\end{array}$ & $\begin{array}{c}q_{1}\left({ }^{7} \mathrm{Be}\right), \\
10^{13}\end{array}$ & $\begin{array}{c}q_{1}\left({ }^{13} \mathrm{~N}\right), \\
10^{13}\end{array}$ & $q_{1}\left({ }^{7} \mathrm{Be}\right) / q_{1}\left({ }^{13} \mathrm{~N}\right)$ & $\begin{array}{c}E_{p x}, \\
\mathrm{keV}\end{array}$ & $\begin{array}{c} \pm \delta E_{p x}, \\
\mathrm{keV}\end{array}$ & $\begin{array}{c}q_{x}\left({ }^{7} \mathrm{Be}\right), \\
10^{13}\end{array}$ & $\begin{array}{c}q_{x}\left({ }^{13} \mathrm{~N}\right), \\
10^{13}\end{array}$ & $\begin{array}{c} \pm \delta q_{x}, \\
10^{13}\end{array}$ \\
\hline 1 & $90(10 /-3)$ & 0.87 & 13 & 0.067 & 550 & 35 & 15.8 & 15.3 & 0.4 \\
2 & Сu-лайнер +10 & 0.02 & 0.58 & 0.035 & 460 & 45 & 0.44 & 1.02 & 0.35 \\
3 & Сu-лайнер+50 & 0.12 & 0.38 & 0.31 & 775 & 25 & 0.41 & 0.40 & 0.1 \\
4 & ПЭ-лайнер+10 & 0.073 & 0.63 & 0.116 & 640 & 30 & 0.71 & 0.66 & 0.1 \\
5 & ПЭ-лайнер+10 & 0.42 & 3.35 & 0.125 & 660 & 30 & 4 & 3.55 & 0.35
\end{tabular}

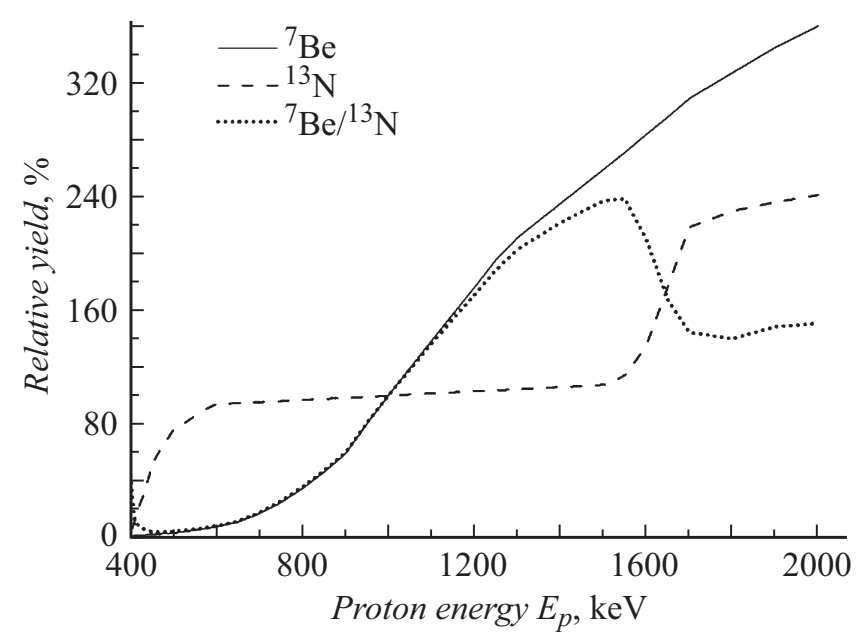

Зависимости относительных выходов реакций ${ }^{10} \mathrm{~B}(p, \alpha){ }^{7} \mathrm{Be}$ и ${ }^{12} \mathrm{C}(p, \gamma){ }^{13} \mathrm{~N}$ от энергии протонов: нормализовано к $100 \%$ при $E_{p}=1000 \mathrm{keV}$.

скотча позволило визуально контролировать отпечатки ионного пучка, не попавшего на мишень $\mathrm{B}_{4} \mathrm{C}$. После облучения каждого образца серией из десяти импульсов, занимавшей в сумме 40-60 s, облученный диск $\mathrm{B}_{4} \mathrm{C}$ немедленно извлекался из вакуумной камеры ускорителя и перемещался на торец коаксиального детектора из высокочистого германия (Canberra) в свинцовой защите для регистрации протонно-индуцированной гаммаактивности ${ }^{7} \mathrm{Be}\left(T_{1 / 2}=53.12\right.$ дня) и ${ }^{13} \mathrm{~N}$ (9.965 min) в пиках с энергией 477.6 и $511 \mathrm{keV}$ соответственно. Типично наведенные активности ${ }^{13} \mathrm{~N}$ регистрировали последовательно по $10 \mathrm{~min}$ трижды, чтобы убедиться в том, что позитронная активность принадлежит исключительно этому радионуклиду, т. е. период полураспада составляет около $10 \mathrm{~min}$. Регистрация наведенной активности ${ }^{7} \mathrm{Be}$ занимала $1-20 \mathrm{~h}$ в зависимости от энергии и флюенсов протонов, перехваченных мишенями карбида бора.

Число протонов $q$, попавших на мишень $\mathrm{B}_{4} \mathrm{C}$, было определено следующим образом:

$$
q \approx N t / e i Y,
$$

где $N$ - интенсивность $\left(\mathrm{s}^{-1}\right)$ гамма-пиков на конец облучения; $t$ - время жизни (s) радионуклидов, определяемое как $T_{1 / 2} / 0.6932 ; e-$ эффективность регистрации гамма-пиков (0.04 для $477.6 \mathrm{keV}$ и 0.03 для $511 \mathrm{keV})$; $i$ - относительная интенсивность гамма-квантов $(0.1052$ для ${ }^{7} \mathrm{Be}$ и 2 для $\left.{ }^{13} \mathrm{~N}\right) ; Y$ выход аналитической реакции (на один протон) для мишени из 100\% ядер ${ }^{10} \mathrm{~B}$ или ${ }^{12} \mathrm{C}$ (например, для протонов с энергией $1 \mathrm{MeV}$ $Y\left({ }^{7} \mathrm{Be}\right) \approx 8.84 \cdot 10^{-6}$ и $\left.Y\left({ }^{13} \mathrm{~N}\right) \approx 7.92 \cdot 10^{-10}\right)$.

В таблице представлены рассчитанные флюенсы и энергии коллективно ускоренных протонов для исследованной геометрии диода Люса. Сначала флюенсы протонов были рассчитаны исходя из предположения, что энергия протонов равна $1 \mathrm{MeV}\left(q_{1}\right)$. Затем находилось отношение выходов обеих реакций $q_{1}\left({ }^{7} \mathrm{Be}\right) / q_{1}\left({ }^{13} \mathrm{~N}\right)$, определяющее действительную энергию протонов $E_{p x}$ (см. рисунок). Окончательно флюенсы протонов корректировались с учетом их действительной энергии до $q_{x}$ умножением флюенсов $q_{1}$ на отношение выходов аналитических радионуклидов при энергии $1 \mathrm{MeV}$ и действительной энергии $E_{p x}$.

Разумно считать, что мишени не эродировали заметно в случаях, когда флюенсы протонов $q_{x}\left({ }^{7} \mathrm{Be}\right)$ и $q_{x}\left({ }^{13} \mathrm{~N}\right)$ имеют сходящиеся значения, например в опытах № 1 и 3-5. Напротив, в опыте № 2 обнаруживаются сильно расходящиеся значения этих флюенсов, что указывает на возможность существенной эрозии поверхности мишени $\mathrm{B}_{4} \mathrm{C}$ при воздействии МИИП в данном случае. Это в свою очередь свидетельствует о том, что энергия протонов была существенно выше, чем измеренная $(460 \mathrm{keV})$. Учитывая, что обе оценки должны взаимно сходиться, энергию протонов в опыте № 2 следует оценивать как $\sim 550 \mathrm{keV}$. В таком случае не обнаруживается увеличения энергии протонов за счет использования медного лайнера на том же расстоянии от анода до мишени, тогда как при дополнительном дистанцировании мишени $\mathrm{B}_{4} \mathrm{C}$ от медного лайнера на +50 mm (опыт № 3) наблюдалось увеличение энергии коллективно ускоренных протонов до $775 \mathrm{keV}$. Увеличение энергии в такой геометрии согласуется с экспериментальными данными об увеличении длины ускорения при оптимизации геометрии эквипотенциальной заанодной области. При применении полиэтиленовых лайнеров (опыты № 4 и 5 ) были обнаружены воспроизводящиеся энергии коллективных ускоренных протонов - около $650 \mathrm{keV}$, что было существенно выше, чем без использования лайнеров.

Нижний предел оцениваемой энергии протонов около $460 \mathrm{keV}$, поскольку выход реакции ${ }^{12} \mathrm{C}(p, \gamma)^{13} \mathrm{~N}$ чрезвычайно мал при энергии существенно ниже резонансной $(459 \mathrm{keV})$. Верхний предел определения энергии протонов с помощью данной методики составляет 
около $1500 \mathrm{keV}$ и предопределяется вторым резонансом $(1698 \mathrm{keV})$ ядерной реакции ${ }^{12} \mathrm{C}(p, \gamma)^{13} \mathrm{~N}$. Для контроля энергий протонов выше $1500 \mathrm{keV}$ нужны мишени, содержащие компонент, на котором протонами возбуждаются реакции с порогом около $1500 \mathrm{keV}$. Так, если карбид ванадия $\mathrm{VC}\left(T_{m}=2800^{\circ} \mathrm{C}\right)$ добавить к матрице карбида бо$\mathrm{pa}$, можно преодолеть ограничение по верхнему пределу энергии протонов за счет регистрации радиоактивности радионуклида ${ }^{51} \mathrm{Cr}\left(T_{1 / 2}=27.7\right.$ дней, $\left.E \gamma=320.1 \mathrm{keV}\right)$, наведенного по реакции ${ }^{51} \mathrm{~V}(p, n){ }^{51} \mathrm{Cr}$, идущей с порогом $1565 \mathrm{keV}$.

Таким образом, развитие предложенной методики видится в приготовлении и тестировании жаропрочной керамики $\mathrm{B}_{4} \mathrm{C}-\mathrm{VC}$, способной выдерживать облучение МИИП с энергиями $1-2 \mathrm{MeV}$ и флюенсами до $(3-5) \cdot 10^{13}$ protons / $\left(\mathrm{cm}^{2} \cdot\right.$ pulse $)$.

\section{Финансирование работы}

Работа выполнена при финансовой поддержке Российского научного фонда (грант № 17-19-01442).

\section{Конфликт интересов}

Авторы заявляют, что у них нет конфликта интересов.

\section{Список литературы}

[1] Luce J.S., Sahlin H., Crites N.R. // IEEE Trans. Nucl. Sci. 1973. V. 20. N 3. P. $336-340$.

[2] Ditrói F., Takács S., Tárkányi F., Fenyvesi A., Bergman J., Heselius S.-J., Solin O. // Nucl. Instrum. Meth. Phys. Res. B. 1995. V. 103. P. 389-392.

[3] Roughton N.A., Fritts M.J., Peterson R.J., Zaidins C.S., Hansen C.J. // Astrophys. J. 1974. V. 188. P. 595-602.

[4] Лопатин В.С., Ремнев Г.Е., Журавлев М.В. // Изв. вузов. Физика. 2018. Т. 61. № 12. С. 121-125.

[5] Howerton R.J., Luce J.S. // Lawrence Livermore Laboratory Report. UCID 16857. August 4, 1975. 